\title{
J. Kochanowski, Treny. Treni. Lamenti, trad. di U. Norsa ed E. Damiani, a cura di G.O. Fasoli, introduz. di L. Marinelli, Agorà \& Co., Lugano 2020, pp. 186.
}

Del più grande poeta rinascimentale polacco nonché uno tra i maggiori del Rinascimento in generale (Jan Kochanowski, I530-1584) sono state recentemente tradotte e pubblicate in italiano due raccolte: le Fraszki, brevi poesie, per la maggior parte epigrammi, tradotte da Nullo Minissi (1995; ristampa del 2002) e le Pieśni, due libri di versi chiaramente ispirati ai Carmina oraziani, pubblicate insieme a una scelta dai Foricoenia (raccolta di epigrammi latini) nella traduzione di Anton Maria Raffo (20ri). Introvabile invece è ormai da anni, al di fuori del circuito delle biblioteche specialistiche, una pregevole traduzione a firma Enrico Damiani dei Treny, ciclo di diciannove elegie polacche in morte della figlia del poeta, Urszula (1926; ristampa del 1930). Uno dei meriti del lavoro di Marinelli e della sua allieva, Giulia Olga Fasoli, è proprio quello di aver reso disponibile anche a un pubblico vasto questo capolavoro della letteratura polacca del Rinascimento. Alla elegante traduzione di Damiani, già edita, i curatori hanno affiancato un testo inedito, ovvero la traduzione prosastica di Umberto Norsa, affascinante figura di schivo e riservato studioso, avvocato di origine ebraica nato nel 1866 e morto nel 1943, mantovano e stimato membro dell'Accademia virgiliana, poliglotta che si è cimentato in traduzioni da lingue le più svariate, tra le quali mette conto ricordare, oltre al greco antico, almeno l'ebraico, il sanscrito e soprattutto l'ungherese (è, quella di Norsa, figura nota ai magiaristi, giusta le sue versioni di Petöfi, Madách e Arany). Le sue numerosissime traduzioni dal polacco risalgono tutte ai decenni che vanno dal 1890 al $1925 \mathrm{e}$ fra di esse si annoverano, oltre ai Treny di Kochanowski, quelle di alcune delle più importanti opere poetiche di Mickiewicz: i Sonetti di Crimea (dati alle stampe da Marinelli nel 1998), l'ancora in gran parte inedita traduzione integrale dei Dziady mickiewiczani (in due versioni; stralci ne furono stampati sul "Fanfulla della Domenica” del I2 agosto e 9 settembre 1894), nonché l'integrale del Pan Tadeusz.

La traduzione prosastica che Fasoli offre per la prima volta al pubblico italiano risale al I893 ed è stampata a fronte del testo polacco, nella colonna di sinistra, mentre nella colonna destra della pagina la curatrice inserisce la traduzione di Damiani. Questa soluzione è assai comoda, giacché consente di avere a immediata disposizione per eventuali raffronti le due traduzioni. Certamente, essa obbliga a forzare la mano, presentando un testo in prosa in forma di versi sciolti, ma in questo caso, come nota lo stesso Marinelli (p. 27), l'operazione trova una convincente giustificazione non solo sul piano dell'immediata utilità pratica, ma anche e forse soprattutto sul piano artistico, in quanto il dettato al contempo semplice ed elegante di Norsa pare spesse volte slanciarsi in direzione di un' ispirata prosa poetica. Del resto, in appendice, Fasoli offre anche la riproduzione fototipica del manoscritto di Norsa a cui affianca la trascrizione prosastica delle traduzioni. 
L'atteggiamento traduttivo di Norsa, da lui stesso discusso in occasione della pubblicazione delle sue traduzioni petöfiane (1913), trova poi importanti consonanze con la temperie culturale in cui l'avvocato mantovano si trovò ad operare: Marinelli ricorda non a caso la posizione di John Middleton Murry il quale, nel suo Classical Translation (1923) argomentò la scelta di una traduzione prosastica atta a liberare il traduttore dai vincoli e lacciuoli che la traduzione poetica gli imporrebbe (rispetto di metro e rime), permettendogli così di essere quanto più possibile fedele all'originale (e proprio il principio di fedeltà al testo ispira evidentemente Norsa). A proposito di Norsa resta infine da dire che la sua lettura dei Treny, così come emerge dalle brevi note introduttive conservateci dal manoscritto e che Fasoli ha trascritto in appendice al volume, è una lettura patentemente romantica, ispirata da quel Lenartowicz che il giovane studente mantovano aveva conosciuto durante la frequenza dei lettorati di polacco tenuti a Bologna da Malwina Ogonowska. Il traduttore nota infatti che i Treny quasi non paiono scritti “in un secolo in cui la voce del cuore raramente uscì schietta fuori dall' animo", aggiungendo poi che nei Treny "i dubbi di un animo pieno di fede e religione [...] sono significati con frasi così tetre come si trovano nei moderni poeti del dolore mondiale [la sottolineatura è di Norsa stesso]” (p. I4I). Se questi sono i presupposti da cui muove Norsa, non dovrà nemmeno stupire l'elegante semplicità del suo dettato ricordata più sopra, giacché egli non faceva altro che accodarsi all'amato Mickiewicz del Cours parigino. Il poeta romantico infatti scorgeva nella pagina dei Treny un tono peculiare alla prosa più semplice che però si trasforma in alta poesia, in un dialogo con la figlia morta che pare assumere una forma epistolare. È a questa scorrevolezza elegante che mira l'italiano della conversazione colta ottocentesca impiegato da Norsa, solo apparentemente semplice. Di questa semplicità peraltro era ben consapevole anche il Damiani, che nella sua traduzione poetica si sforzerà di conservare all'opera un tono piano e finanche spontaneo, insomma mimetico del dialogato elegante.

La versione del più giovane Enrico Damiani (1892-1953) nasce invece poetica, in versi e appare meglio riuscita e di maggiore accortezza rispetto a quella del Norsa, che avrà pagato anche una certa inesperienza (tradusse la raccolta senza avere alle spalle il bagaglio di letture di Damiani); la traduzione di Norsa non è tuttavia priva di momenti notevoli come quando, traducendo il primo lamento (tren, che Norsa traduce con il grecismo 'treno') mostra di cogliere pienamente il valore espressivo dell'anafora di wszytki, mimetico del pianto singhiozzante; se nel primo testo della raccolta entrambi i traduttori colgono il valore di questa anafora nonché la ricorsività finanche tematica dell'avverbio próżno, nel caso del lamento IX 3 Norsa si dimostra più efficace di Damiani, poiché traduce ai vv. I-3: "O saggezza [...] [che] sai sradicare [wykorzenić] dall'animo tutte le brame”, laddove Damiani sceglie questa resa: "che puoi [...] qualunque sofferenza / umana o desiderio placare [...]". La scelta di Norsa lo costringe ad aggiungere il sintagma "dell'animo", assente nell'originale polacco, ma al contempo gli consente di essere più aderente al dettato kochanoviano. Questa fedeltà al testo è tanto più apprezzabile in quanto dietro al verbo wykorzenić, come riporta Fasoli sulla scorta di Mayenowa e Woronczakowa, si nascondono dei passi ciceroniani (Tusc. II 5, I3: extrahit [philosophia] vitia radicitus; IV 38, 83: errorem quasi radicem [...] stirpitus philosophia se extracturam pollicetur). Un atteggiamento 'conservativo' nei confronti della lettera dell'originale è tanto più apprezzabile oggi che il poderoso commento di Mayenowa e Woronczakowa uscito nel 1983 per l'edizione sejmowa delle opere kochanoviane ci ha ormai resi consapevoli (ciò che ancora né Norsa né Damiani potevano essere) della ricchissima bumus classica sulla quale sono cresciuti rigogliosi i Treny. Del resto non sempre le scelte che Damiani vorrebbe poetiche risultano azzeccate e Fasoli nota giustamente che la traduzione di Treny Xv 3 (porzucić dusze) proposta da Damiani è francamente brutta ("debbo l'anima gettare"), mentre quella di Norsa, più letterale ("debbo abbandonare l'anima”) risulta senz'altro più riuscita. Si farebbe però un torto a Damiani se non si segnalasse almeno uno (ma non l'unico!) luogo in cui è la sua traduzione a essere superiore a 
quella di Norsa (che paradossalmente proprio nel luogo di cui si dirà, abbandona il principio di fedeltà al testo); la splendida iunctura "rack tamania" (lett.: 'il rompersi delle mani', da immaginarsi come movimento continuato, ossessivo e violento delle mani del piangente), viene reso dal Norsa con "atti di disperazione", mentre Damiani, più efficacemente, opta per i “contorcimenti delle mani”.

Il commento approntato da Fasoli (che integra le note, invero abbastanza essenziali, di Damiani e Norsa), è estremamente interessante sia per l'attento studio comparato delle due traduzioni, sia per la raccolta di loci paralleli, cha spaziano dai testi sacri alla letteratura mediolatina (Petrarca e Boccaccio latini), passando per i testi biblici. Ora, molti di questi loci provengono dall'editio maior di Mayenowa e Woronczakowa e la curatrice lo dichiara, eppure è d'obbligo sottolineare il suo intelligente lavoro critico; la studiosa infatti, sfrondando per così dire le esuberanti chiome dell'edizione polacca, è riuscita a procurare un apparato di commento sintetico ma che al contempo non sacrifica nessuno degli elementi essenziali per comprendere e apprezzare il retroterra culturale dei Treny: Cicerone, tra i latini citati, è molto presente, ma non mancano abbondanti paralleli con Orazio, Virgilio e Stazio; talvolta fanno capolino anche gli elegiaci o gli epigrammisti della Planudea, né mancano paralleli con i testi biblici. I loci paralleli proposti nel commento comprendono anche il Petrarca e il Boccaccio latini e colpisce in particolare la ricorrenza del Petrarca latino, spesso indiziato di essere scaturigine prima di determinate iuncturae (si veda anche solo il caso di "impia mors" in Treny I 7), sospetto forte anche nelle elegie latine del poeta (è il caso ad esempio di "irrediturus" in II 3, 24, mediolatinismo attestato, prima di Petrarca, solo in testi difficilmente letti da Kochanowski come ad esempio i Gesta Danorum di Saxo Germanicus).

Va infine segnalata l'importante Introduzione di Luigi Marinelli, soprattutto per la prima parte, che è un notevole studio sulla struttura numerologica architettata dal poeta. Il numero di componimenti (19), apparentemente incongruo rispetto alla tradizione dei cicli poetici, viene spiegato alla luce delle interpretazioni numerologiche offerte dalla patristica nonché dall'esegesi medievale (Ugo da San Vittore) e dalla trattatistica rinascimentale (Pietro Bongo, De Mystica numerorum significatione). L'idea di fondo di Marinelli, ben argomentata e poggiata su solidi riscontri è che il 19 , nella sua imperfezione, racchiuda in sé l'elemento umano (imperfetto, appunto) e l'elemento divino, rappresentato dall' $\mathrm{I}$, anch'esso presente nello stesso $\mathrm{I} 9(\mathrm{I}+9=\mathrm{IO} \rightarrow \mathrm{I}+\mathrm{O}=\mathrm{I})$. Una volta scomposto il $\mathrm{I} 9$ nel modo appena presentato sarà allora chiaro come un passaggio del De scripturis et scriptoribus sacris di Ugo da San Vittore aiuti a spiegare l'elemento umano presente in questo numero giacché, secondo il dotto medievale, novenarius ante denarium [significat] defectum intra perfectionem (pp. 12-13; I6). Sono poi notevoli i paralleli che Marinelli individua tra formulazioni presenti nei singoli treny e alcune che si riscontrano nelle traduzioni polacche dei salmi firmate dallo stesso Kochanowski (si veda ad esempio la rappresentazione del sole eterno della divinità in Treny XIX 73-74 e in Psalmy XIX 27-28). Questi paralleli all' interno dell'opera kochanoviana, assai convincenti, suggeriscono nuovi indirizzi di ricerca, che portino allo studio dell'esegesi dei testi biblici e dei significati che i Padri della Chiesa prima e poi i dotti medievali e rinascimentali attribuivano a determinati numeri. Marinelli per primo scrive che non necessariamente proprio e solo quei testi dell'esegesi medievale e della numerologia rinascimentale da lui proposti dovevano essere noti a Kochanowski (è quasi certamente il caso del De Mystica di Pietro Bongo, databile al 1583 -1584, ovvero a ridosso della morte del poeta e quindi difficilmente da lui compulsabile), ma il suo lavoro alla traduzione dei salmi poteva benissimo avergli fatto incontrare testi assimilabili a quelli allineati dallo studioso; ciò che conta è ad ogni modo l'ipotesi di ricerca: la strada tracciata è senz' altro convincente e degna di futuri approfondimenti. 